\title{
Prácticas seguras en administración de medicamentos en un hospital de segundo nivel de atención
}

\author{
María-Alejandra Ortega-Barco ${ }^{1,}$ * y Daniela Marín-Rodríguez ${ }^{2}$ \\ 1 Enfermera, Magíster en Enfermería, Docente del Programa de Enfermería de la Fundación Universitaria de \\ San Gil, Santander, Colombia; e-mail@e-mail.com; ORCID id: https://orcid.org/0000-0002-6851-066X \\ 2 Enfermera, Secretaría de Salud del departamento de Santander, Colombia; ORCID id: \\ https://orcid.org/0000-0001-5970-7498 \\ * Autor correspondencia: mariaortega@unisangil.edu.co
}

DOI: https://doi.org/10.37536/RIECS.2021.6.2.276

Recibido: 26/08/2021; Aceptado: 25/10/2021; Publicado: 30/11/2021

Resumen: Las prácticas de administración segura de medicamentos hacen parte importante del compendio de actividades para resguardar la seguridad del paciente, por tanto, la búsqueda de un entorno seguro es una preocupación constante de las instituciones de salud, y la práctica de la administración de medicamentos es esencial para evitar errores de medicación. La medición de las prácticas del personal de enfermería respecto a la administración de medicamentos puede generar datos relevantes para la elaboración de estrategias más fidedignas de la necesidad institucional y en consecuencia disminuir notablemente la aparición de errores de medicación. Objetivo: Determinar el cumplimiento de prácticas en administración segura de medicamentos por parte del personal de enfermería de un hospital de segundo nivel de atención. Metodología: Estudio descriptivo transversal en el que se evaluó el grado de cumplimiento de prácticas de administración segura de medicamentos a través de la aplicación de dos listas de chequeo. Resultados: El ejercicio de prácticas de seguridad en administración de medicamentos en este estudio mostró un rendimiento del $61 \%$ en personal técnico auxiliar de enfermería y un $49 \%$ en profesionales de enfermería, con Prevalencia de no cumplimiento del $27,9 \%$ y $42,9 \%$, respectivamente. Coeficiente de correlación de Spearman 0,71 en los resultados de las listas de chequeo. Conclusiones: El personal de enfermería, en este estudio, mostró cumplimiento de prácticas seguras en administración de medicamentos alrededor de la media de cumplimiento, con un rendimiento similar en ambos grupos del personal de enfermería y en ambas listas de chequeo.

Palabras Clave: Utilización de medicamentos, Atención de enfermería, Seguridad del paciente, Servicios de enfermería, Personal de Enfermería.

\begin{abstract}
Safe drug administration practices are an important part of the compendium of activities to protect patient safety, therefore, the search for a safe environment is a constant concern of health institutions, and the practice of drug administration is essential to avoid medication errors. The measurement of the nursing staff's practices regarding the administration of medications can generate relevant data for the elaboration of more reliable strategies of the institutional need and consequently significantly reduce the appearance of medication errors. Objective: To determine compliance with practices in the safe administration of medications by the nursing staff of a second level of care hospital. Methodology: A descriptive cross-sectional study in which the degree of compliance with safe drug administration practices was evaluated through the application of two checklists. Results: The exercise of safety practices in drug administration in this study showed a performance of $61 \%$ in nursing auxiliary technical personnel and $49 \%$ in nursing professionals, with a prevalence of non-compliance of $27.9 \%$ and $42.9 \%$, respectively. Spearman's correlation coefficient 0.71 in the checklist results. Conclusions: The nursing staff, in this study, showed compliance with safe practices in drug administration around the mean of compliance, with a similar performance in both groups of the nursing staff and in both checklists.
\end{abstract}


Key words: Drug utilization, Nursing care, Patient safety, Nursing services, Nursing Staff.

\section{Introducción}

La administración segura de medicamentos es un tema principal que la Organización Mundial de la Salud (OMS) tiene en cuenta, ya que los errores más perjudiciales están relacionados con el diagnóstico, prescripción y uso de medicamentos [1], y a su vez invita a que nadie debiera sufrir daños por la atención en salud, ya que 3\% de las personas desarrolla evento adverso debido a medicación [2], 23,9\% sufre reacciones adversas graves a los medicamentos [3] y se estima que 2,6 millones de personas mueren cada año [1] por daños que pudieron ser evitados.

En la administración segura de medicamentos el personal de enfermería (PE) desempeña un papel trascendental como último eslabón en la gestión integral del medicamento hasta la recepción por el paciente, los errores de medicamentos (EM) más frecuentes ocurren en las etapas de la prescripción y administración [4], de los cuales 50-70\% se consideran errores prevenibles [5] y corresponden la mitad de ellos, a la disposición que el PE realiza de dichos medicamentos.

En respuesta a ello, el Ministerio de Salud de Colombia ha desarrollado estrategias para alinear los requisitos en los programas de seguridad del paciente y disminuir la incidencia de EM, que las instituciones de salud en general han adoptado en sus protocolos para disminuir la aparición de dichos eventos. No obstante, los EM y sus consecuencias negativas, siguen constituyéndose en la principal causa de eventos adversos en los hospitales, representando el 19,4\% del total de lesiones que producen discapacidad o muerte [5]; y a su vez, el $25 \%$ de los eventos adversos que se producen durante la administración de medicamentos (AM) se deben a falta de conocimientos en relación con el fármaco [6] y el 17\% se deben a falta de información en relación con el paciente [5].

Lo anterior se traduce también en un problema para la institución y el sistema de salud, ya que además de los resultados de salud deficientes en la población, los medicamentos y otras tecnologías representan el porcentaje más alto del costo del tratamiento y la atención [7] el cual incrementa a mayor número de errores, y en instituciones más grandes con mayor flujo de pacientes.

A pesar de las estrategias de mitigación y control, los EM siguen ocurriendo frecuentemente por la omisión y fallas en los correctos, las interrupciones, distracciones, carga laboral, estrés [8], falta de conocimiento y comunicación ineficaz [2]; además, el desconocimiento farmacológico por parte de del PE es un componente importante en la AM errónea [9], ya que es quién debe tener las competencias para desarrollar la actividad correctamente, poseer conocimientos fisiológicos y farmacológicos, y habilidades necesarias asociadas a la administración para contribuir a la seguridad del paciente.

La búsqueda de un entorno seguro es una preocupación constante de las instituciones de salud $[2,10]$, no obstante, el personal de salud no se adhiere a lineamientos institucionales si no encuentra convicciones profundas para hacerlo y la práctica de dichos protocolos se logra con el reforzamiento continuo de los criterios de seguridad en la AM. La adherencia a los protocolos y guías institucionales es esencial para identificar los errores en la atención, generar planes de mejoramiento y optimizar los resultados de salud [11], sin embargo, el conocimiento de los mismos no está garantizando su cumplimiento y ejecución [12] y se requieren estrategias enfocadas al problema detectado.

La medición de prácticas del PE respecto a la AM puede generar datos relevantes para la elaboración de estrategias más fidedignas de la necesidad institucional y en consecuencia disminuir notablemente la aparición de EM con afectación a la seguridad del paciente. El objetivo de este estudio fue determinar el cumplimiento de prácticas en administración segura de medicamentos por parte del personal de enfermería de un hospital de segundo nivel de atención. 


\section{Metodología}

Estudio descriptivo transversal en el que se evaluó el grado de cumplimiento de las prácticas de administración segura de medicamentos a través de la aplicación de dos listas de chequeo.

La muestra fue a conveniencia dado que se tomaron 14 días en los que un evaluador externo, profesional de enfermería, aplicó las listas de chequeo al PE (profesional o técnico auxiliar de enfermería) que se encontrara de turno en dicho día en un servicio asistencial. El diligenciamiento de las listas se realizó a través de la observación del proceso de AM con registro virtual (en la nube). Se recogieron datos de un total de 50 integrantes del PE de los servicios de hospitalización del Hospital de segundo nivel de atención en Santander (Colombia), en el periodo de junio a julio de 2021; posteriormente se diligenciaron datos sociodemográficos y de formación académica, además de datos básicos relacionados con la administración segura de medicamentos.

La aplicación de las listas de chequeo fue aprobada por el Comité de ética de la Fundación Universitaria de San Gil y por la administración del Hospital de segundo nivel. Se informó al personal de la ocurrencia de este proyecto de investigación y 75 integrantes del PE firmaron el consentimiento informado. Prevaleció el respeto por la dignidad de los integrantes y el respeto de sus derechos, se tuvo en cuenta los principios éticos contemplados en la Ley 911 de 2004: Beneficencia, No maleficencia, Justicia, Autonomía, Fidelidad y Reciprocidad; y el estudio fue clasificado como de riesgo mínimo y se protegió la confidencialidad de información de los participantes.

Las listas de chequeo aplicadas fueron las siguientes:

1. Lista de chequeo del procedimiento para la administración de medicamentos vía oral, intradérmica, subcutánea, intramuscular e intravenosa creada a partir del conocimiento consignado en el Manual de administración segura de medicamentos de la Universidad del Bosque (Colombia, 2018) [13]; que cuenta con 30 enunciados para marcar el cumplimiento por parte del PE durante el proceso de AM.

2. Lista de chequeo tomada del Instrumento para la administración segura de medicamentos a través de los 10 correctos (Rebouças et al, Brasil, 2018) [14], que reporta Alfa de Cronbach de 0,85; y cuenta con 27 enunciados, relacionado con los 10 correctos de la medicación, para marcar el cumplimiento por parte del PE durante el proceso de AM.

Se realizó el proceso de validez facial y de contenido de las dos listas de chequeo, en el que participaron 25 expertos, quienes realizaron la evaluación diligenciando un formato creado por el PhD Fabio Camargo ${ }^{1}$, el cual evalúa la validez facial a través de la comprensión, claridad y precisión del ítem y la validez de contenido a través de la pertinencia y relevancia del ítem en una escala tipo Likert de puntaje entre 1 y 5. Se utilizó la técnica del Modelo de Fehring y se determinó el Índice de Acuerdo (IA) y el Índice de validez de contenido (IVC), se descartaron los ítems que no alcanzaron IVC de 0,8 y se adecuaron los instrumentos.

El instrumento Lista de chequeo del procedimiento para la administración de medicamentos vía oral, intradérmica, subcutánea, intramuscular e intravenosa construido, alcanzó IA de 0,98 para comprensión, 0,98 para claridad, 0,98 para precisión, e IVC de 0,98 para pertinencia y 0,99 para relevancia. Finalmente, este instrumento reporta validez con IA de 0,98 e IVC de 0,99. Ver Tabla I.

El instrumento Valoración de la seguridad del paciente en la administración de medicamentos fue tomado del estudio Medication administration safety assessment tool: construction and validation de Rebouças et al (Brasil, 2018) [14] y traducido al español latino. Alcanzó IVC de 0,77 para simplicidad, 0,76 para claridad y 0,93 para relevancia en su país de creación. El análisis factorial exploratorio se mostró adecuado, el AC fue de 0,85. Después de ser traducido al español, en Colombia, pasó por proceso de validez facial y alcanzó IA de 0,98 para comprensión, 0,98 para claridad, 0,98 para precisión; se realizó edición de la traducción del documento según lo aportado por expertos y se adecuó versión final. Finalmente, este instrumento reporta validez con IVC de 0,82 y confiabilidad

\footnotetext{
${ }^{1}$ Enfermero, Magíster en Epidemiología, Doctor en Epidemiología. Proporcionó el formato de validez facial y de contenido de su creación para aplicar en el proceso de validación de los instrumentos de este estudio
} 
con AC de 0,85 determinados en su país de origen (Brasil, 2018) y validez en su traducción al español en Colombia $(2020)^{2}$, con IA de 0,98 . Ver Tabla I.

Tabla I Validez y confiabilidad para los instrumentos

\begin{tabular}{|c|c|c|c|c|c|c|c|c|c|}
\hline \multirow[b]{2}{*}{$\begin{array}{c}\text { Dimensión } \\
\text { CAP }\end{array}$} & \multirow[b]{2}{*}{ Instrumento } & \multicolumn{4}{|c|}{ Validez facial } & \multicolumn{3}{|c|}{ Validez de contenido } & \multirow{2}{*}{$\begin{array}{c}\text { Confiabilidad } \\
\text { Alfa de } \\
\text { Cronbach }\end{array}$} \\
\hline & & Comprensión & Claridad & Precisión & IA & Pertinencia & Relevancia & IVC & \\
\hline \multirow[t]{2}{*}{ 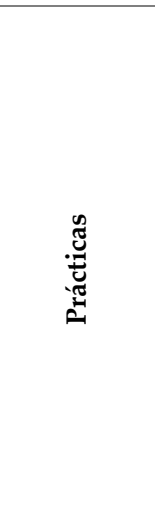 } & $\begin{array}{l}\text { Lista de chequeo } \\
\text { del procedimiento } \\
\text { para la } \\
\text { administración de } \\
\text { medicamentos vía } \\
\text { oral, intradérmica, } \\
\text { subcutánea, } \\
\text { intramuscular e } \\
\text { intravenosa }\end{array}$ & 0,98 & 0,98 & 0,98 & 0,98 & 0,98 & 0,99 & 0,99 & - \\
\hline & $\begin{array}{l}\text { Valoración de la } \\
\text { seguridad del } \\
\text { paciente en la } \\
\text { administración de } \\
\text { medicamentos }\end{array}$ & 0,98 & 0,98 & 0,98 & 0,98 & - & - & $0,82^{*}$ & $0,85^{*}$ \\
\hline
\end{tabular}

\section{Resultados}

El Hospital de segundo nivel cuenta con 114 integrantes del PE (19 profesionales y 95 técnicos auxiliares); posterior a la aprobación del proyecto 75 integrantes firmaron el consentimiento informado para la participación dentro del estudio, 50 de ellos recibieron la aplicación de las dos listas de chequeo por parte de un evaluador externo y la determinación de datos sociodemográficos y de formación académica.

\subsection{Características sociodemográficas}

El 14\% de los participantes eran profesionales de enfermería y el $86 \%$ restante eran técnicos auxiliares de enfermería. Respecto a las edades, el rango de edad predominante fue de 26 a 30 años, $42,8 \%$ en los profesionales y $30,2 \%$ en los técnicos auxiliares. Los profesionales cuentan con experiencia en área asistencial de 4 a 5 años o de16 a 20 años (ambos rangos: 28,5\%); para los técnicos auxiliares, el 39,5\% cuenta con 6 a 10 años de experiencia en el área asistencial, solo el 14,3\% (un profesional) manifestó tener menos de 1 año de experiencia en área asistencial y para el caso de los técnicos auxiliares este porcentaje fue del $7 \%$. El servicio asistencial en el que la mayoría de los integrantes ha laborado por más tiempo es el servicio de Urgencias o UCI, $42,9 \%$ de los profesionales y $76,8 \%$ de los técnicos auxiliares.

El 28,6\% de los profesionales evaluados manifiesta haber realizado formación posgradual y el $7 \%$ de los técnicos auxiliares dice haberse formado como regente de farmacia. En cuanto a cursos de actualización en administración segura de medicamentos fuera del lugar de trabajo, solo el $28,5 \%$ de los profesionales y el $37,2 \%$ de los técnicos auxiliares manifiesta haber recibido dicha formación. En cuanto a otras actualizaciones en el lugar de trabajo, el 57,1\% de los profesionales y el $70 \%$ de los técnicos auxiliares dice haber recibido formación en farmacología; para la formación en administración segura de medicamentos estos porcentajes ascienden a $71,4 \%$ en los profesionales y $74,4 \%$ en los técnicos auxiliares.

El $28,6 \%$ de los profesionales definieron su nivel de conocimiento farmacológico de los medicamentos utilizados en su unidad de trabajo como Ideal/Muy satisfactorio; el 57,1\% como Bueno/Satisfactorio y el 14,3\% como Malo/Insatisfactorio. Para el caso de los técnicos auxiliares, estos 
porcentajes se distribuyeron así, 30,2\% en conocimiento Ideal/Muy satisfactorio, $69,8 \%$ como Bueno/Satisfactorio y ningún técnico auxiliar definió su conocimiento farmacológico como Malo/Insatisfactorio.

Para el caso de autopercepción del nivel de conocimientos en administración segura de medicamentos, el 42,8\% de los profesionales definieron su nivel de conocimiento como Ideal/Muy satisfactorio, igual porcentaje para nivel de conocimiento Bueno/Satisfactorio y el 14,3\% como Malo/Insatisfactorio. Para el caso de los técnicos auxiliares, 32,6\% definieron su conocimiento en administración segura de los medicamentos como Ideal/Muy satisfactorio, 67,4\% como Bueno/Satisfactorio y ningún técnico auxiliar definió su conocimiento como Malo/Insatisfactorio. Ver Tabla II.

El 77,3\% de los evaluados manifiesta que existe un protocolo, manual o guía para preparar, diluir y administrar medicamentos en su unidad de trabajo y $74,2 \%$ manifiestan que hubo capacitación para el uso de dicho protocolo, manual o guía.

Tabla II. Datos sociodemográficos y de formación por grupos profesionales del personal de enfermería

\begin{tabular}{|c|c|c|c|c|}
\hline \multirow[b]{3}{*}{ Variable } & \multicolumn{4}{|c|}{ Grupo } \\
\hline & \multicolumn{2}{|c|}{$\begin{array}{l}\text { Profesionales } \\
\qquad(n=7)\end{array}$} & \multicolumn{2}{|c|}{$\begin{array}{l}\text { Técnicos auxiliares } \\
(n=43)\end{array}$} \\
\hline & Número & $\%$ & Número & $\%$ \\
\hline \multicolumn{5}{|l|}{ Datos socio demográficos y laborales } \\
\hline \multicolumn{5}{|l|}{ Grupo de edad en años } \\
\hline $21-25$ & 0 & 0,0 & 8 & 18,6 \\
\hline $26-30$ & 3 & 42,8 & 13 & 30,2 \\
\hline $31-35$ & 2 & 28,6 & 6 & 14,0 \\
\hline $36-40$ & 2 & 28,6 & 6 & 14,0 \\
\hline $41-45$ & 0 & 0,0 & 4 & 9,3 \\
\hline $46-50$ & 0 & 0,0 & 4 & 9,3 \\
\hline $51-55$ & 0 & 0,0 & 1 & 2,3 \\
\hline 56 y más & 0 & 0,0 & 1 & 2,3 \\
\hline \multicolumn{5}{|l|}{ Tiempo de experiencia en años } \\
\hline Menor a 1 & 1 & 14,3 & 3 & 7,0 \\
\hline 1 a 3 & 1 & 14,3 & 8 & 18,6 \\
\hline 4 a 5 & 2 & 28,5 & 3 & 7,0 \\
\hline 6 a 10 & 1 & 14,3 & 17 & 39,5 \\
\hline 11 a 15 & 0 & 0,0 & 5 & 11,6 \\
\hline 16 a 20 & 2 & 28,5 & 4 & 9,3 \\
\hline Mayor a 20 & 0 & 0,0 & 3 & 7,0 \\
\hline \multicolumn{5}{|l|}{ Servicio donde ha laborado la mayor parte del tiempo } \\
\hline Urgencias o UCI & 3 & 42,9 & 33 & 76,8 \\
\hline Otro servicio de hospitalización & 3 & 42,9 & 5 & 11,6 \\
\hline Otro servicio fuera de hospitalización & 1 & 14,2 & 5 & 11,6 \\
\hline \multicolumn{5}{|l|}{ Datos de Formación académica y otros } \\
\hline Formación adicional & Posgrado & & $\begin{array}{l}\text { Regente } \\
\text { farmacia }\end{array}$ & \\
\hline Sí & 2 & 28,6 & 3 & 7,0 \\
\hline No & 5 & 71,4 & 40 & 93,0 \\
\hline \multicolumn{5}{|l|}{$\begin{array}{l}\text { Curso de actualización en Administración segura de } \\
\text { medicamentos }\end{array}$} \\
\hline Sí & 2 & 28,5 & 16 & 37,2 \\
\hline No & 5 & 71,5 & 27 & 62,8 \\
\hline \multicolumn{5}{|l|}{ Formación en farmacología en el lugar de trabajo } \\
\hline Sí & 4 & 57,1 & 30 & 70,0 \\
\hline No & 3 & 42,9 & 13 & 30,0 \\
\hline \multicolumn{5}{|l|}{$\begin{array}{l}\text { Formación en administración segura de medicamentos } \\
\text { en el lugar de trabajo }\end{array}$} \\
\hline Sí & 5 & 71,4 & 32 & 74,4 \\
\hline No & 2 & 28,6 & 11 & 25,6 \\
\hline \multicolumn{5}{|l|}{$\begin{array}{l}\text { Autopercepción del nivel de conocimientos en } \\
\text { farmacología }\end{array}$} \\
\hline Ideal/Muy satisfactorio & 2 & 28,6 & 13 & 30,2 \\
\hline
\end{tabular}




\begin{tabular}{ccccc}
\hline Bueno/Satisfactorio & 4 & 57,1 & 30 & 69,8 \\
\hline Malo/Insatisfactorio & 1 & 14,3 & 0 & 0,0 \\
\hline $\begin{array}{l}\text { Autopercepción del nivel de conocimientos } \\
\text { administración segura de medicamentos }\end{array}$ & & & & \\
\hline Ideal/Muy satisfactorio & 3 & 42,8 & 14 & 32,6 \\
\hline Bueno/Satisfactorio & 3 & 42,8 & 29 & 67,4 \\
\hline Malo/Insatisfactorio & 1 & 14,3 & 0 & 0,0 \\
\hline
\end{tabular}

Fuente: Autoras

\subsection{Resultados en las listas de chequeo de Prácticas en la administración segura de medicamentos}

3.2.1. Lista de chequeo del procedimiento para la administración de medicamentos vía oral, intradérmica, subcutánea, intramuscular e intravenosa

En promedio, el 29,5\% del PE no cumple con las prácticas de seguridad en la AM evaluadas, siendo Lavado clínico de manos previo a la AM, Valoración del paciente (información general y antecedentes), Valoración física del paciente, Evaluación de datos de laboratorio, Análisis de signos vitales, Lavado clínico de manos posterior a la AM, Valoración del paciente en busca de reacciones adversas a la medicación, las prácticas en la AM que más del 50\% del PE no cumple. Las prácticas de seguridad que más del 90\% del PE cumple son: Alistar equipo completo antes de la AM, Eliminar aire de la jeringa, Administrar medicamento teniendo en cuenta precauciones y técnica según vía de administración, Disposición de los desechos en las canecas correspondientes. Ver Tabla III.

Tabla III Prácticas de seguridad del personal de enfermería durante la administración de medicamentos

\begin{tabular}{|c|c|c|c|}
\hline ACTIVIDADES & SÍ & NO & NA \\
\hline Realizó el lavado clínico de manos previo a la administración de medicamentos & $36 \%$ & $64 \%$ & \\
\hline Utilizó elementos de protección personal de acuerdo a la situación clínica del paciente & $76 \%$ & $24 \%$ & \\
\hline $\begin{array}{l}\text { 3. Verificó los } 10 \text { correctos: } \\
\text { Verificó } 10 \text { correctos } \\
\text { Verificó } 9 \text { correctos } \\
\text { Verificó } 8 \text { correctos } \\
\text { Verificó } 7 \text { correctos } \\
\text { Verificó } 6 \text { correctos }\end{array}$ & $\begin{array}{l}34 \% \\
8 \% \\
22 \% \\
26 \% \\
10 \%\end{array}$ & & \\
\hline $\begin{array}{l}\text { 4. Realizó la valoración del paciente teniendo en cuenta información general y antecedentes } \\
\text { personales y familiares }\end{array}$ & $36 \%$ & $60 \%$ & $4 \%$ \\
\hline $\begin{array}{l}\text { 5. Realizó la valoración física utilizando: inspección, palpación, percusión y auscultación, si } \\
\text { procedía. (Valoró presencia de náuseas o vómito, disfagia, diarrea, peristaltismo, así mismo } \\
\text { estado de conciencia, capacidad de comprensión y de colaboración, glositis, estomatitis, } \\
\text { limitaciones físicas o psíquicas; Valoración de pares craneales glosofaríngeo e hipogloso, mucosa } \\
\text { oral, motilidad de la lengua, capacidad para movilizar el cuello, para el caso de medicamento } \\
\text { vía oral) }\end{array}$ & $0 \%$ & $66 \%$ & $34 \%$ \\
\hline $\begin{array}{l}\text { 6. Evaluó datos de laboratorio como pruebas de función renal y hepática, parcial de orina, cuadro } \\
\text { hemático, pruebas de coagulación, electrolitos, función tiroidea, si procedía }\end{array}$ & $0 \%$ & $68 \%$ & $32 \%$ \\
\hline Analizó signos vitales & $16 \%$ & $84 \%$ & \\
\hline Alistó el equipo completo de administración de medicamentos según era el caso & $96 \%$ & $4 \%$ & \\
\hline 9. $\quad$ Se colocó los guantes limpios durante todo el proceso & $76 \%$ & $24 \%$ & \\
\hline $\begin{array}{l}\text { 10. Envasó el medicamento aspirando con la jeringa la totalidad del fármaco sin tocar los bordes de } \\
\text { la ampolla }\end{array}$ & $72 \%$ & $18 \%$ & $10 \%$ \\
\hline 11. En caso de vial, desinfectó el tapón & $14 \%$ & $2 \%$ & $84 \%$ \\
\hline 12. Reconstituyó y envasó conservando la técnica aséptica & $29 \%$ & $4 \%$ & $38 \%$ \\
\hline $\begin{array}{l}\text { 17. Realizó la desinfección del puerto del equipo, o del buretrol o del tapón por donde administró } \\
\text { el medicamento }\end{array}$ & $62 \%$ & $20 \%$ & $18 \%$ \\
\hline $\begin{array}{l}\text { 18. Colocó o asistió al paciente para adoptar la posición más conveniente de acuerdo a la zona donde } \\
\text { aplicó el medicamento }\end{array}$ & $86 \%$ & $14 \%$ & \\
\hline 19. Determinó la zona de administración, para el caso de medicamento intramuscular o subcutáneo & $16 \%$ & $0 \%$ & $84 \%$ \\
\hline
\end{tabular}




\begin{tabular}{|c|c|c|c|c|}
\hline & $\begin{array}{l}\text { Realizó la limpieza de la piel adecuadamente, para el caso de medicamento intramuscular, } \\
\text { subcutáneo o intradérmico }\end{array}$ & $14 \%$ & $0 \%$ & $86 \%$ \\
\hline 21. & $\begin{array}{l}\text { Evitó tocar la tableta, gragea o cápsula directamente con los dedos al administrar medicamentos } \\
\text { vía oral }\end{array}$ & $8 \%$ & $2 \%$ & $90 \%$ \\
\hline 22. & $\begin{array}{l}\text { En administración de medicamento por vía oral, ofreció la medicina y el agua al paciente y } \\
\text { verificó que la haya ingerido }\end{array}$ & $2 \%$ & $8 \%$ & $90 \%$ \\
\hline 23. & $\begin{array}{l}\text { Administró el medicamento teniendo en cuenta las precauciones y técnica según la vía de } \\
\text { administración }\end{array}$ & $94 \%$ & $6 \%$ & \\
\hline 24. & Dispuso los desechos en las canecas y recipientes destinados para cada uno de ellos & $90 \%$ & $10 \%$ & \\
\hline 25. & Descartó agujas sin re enfundar en el guardián o recipiente según normas de bioseguridad & $52 \%$ & $44 \%$ & $4 \%$ \\
\hline 26. & Dejó al paciente cómodo manteniendo las medidas de seguridad & $86 \%$ & $14 \%$ & \\
\hline 27. & Realizó lavado clínico de manos posterior a la realización de la administración de medicamentos & $20 \%$ & $80 \%$ & \\
\hline 28. & Después de la administración valoró al paciente periódicamente en busca de reacciones adversas & $28 \%$ & $72 \%$ & \\
\hline 29. & $\begin{array}{l}\text { En el caso de administración intradérmica, a los 20-30 minutos valoró la pápula, mide tamaño y } \\
\text { comparó con induración de la inyección }\end{array}$ & $0 \%$ & $8 \%$ & $92 \%$ \\
\hline & $\begin{array}{l}\text { Elaboró el registro teniendo en cuenta: datos de identificación del paciente, fecha, hora, } \\
\text { medicamento, dosis, vía, nombre de quién administró el medicamento, tolerancia, reacciones } \\
\text { presentadas y otras observaciones que consideró pertinentes }\end{array}$ & $58 \%$ & $42 \%$ & \\
\hline
\end{tabular}

\section{Fuente: Autoras}

Es importante denotar que, el $72 \%$ del PE no realizó el lavado clínico de manos para el proceso de AM (antes y después), el 70\% no realizó valoración al paciente antes y después de la AM, el 66\% no revisó los correctos de la medicación en su totalidad, el $42 \%$ no elaboró los registros correspondientes y el 36\% no explicó el procedimiento ni informó al paciente sobre el proceso.

El cumplimiento de las prácticas de administración segura de medicamentos por rol profesional se dio así (ver Tabla IV):

Tabla IV Cumplimiento de las prácticas de seguridad del personal de enfermería durante la administración de medicamentos por rol profesional

\begin{tabular}{|c|c|c|}
\hline ACTIVIDADES & 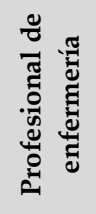 & 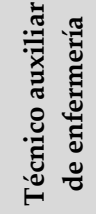 \\
\hline Realizó el lavado clínico de manos previo a la administración de medicamentos & $42,9 \%$ & $34,9 \%$ \\
\hline Utilizó elementos de protección personal de acuerdo a la situación clínica del paciente & $57,1 \%$ & $79,1 \%$ \\
\hline Verificó los 10 correctos & $14,3 \%$ & $37,2 \%$ \\
\hline $\begin{array}{l}\text { 4. Realizó la valoración del paciente teniendo en cuenta información general y antecedentes personales } \\
\text { y familiares }\end{array}$ & $0 \%$ & $46,6 \%$ \\
\hline $\begin{array}{l}\text { 5. Realizó la valoración física utilizando: inspección, palpación, percusión y auscultación, si procedía. } \\
\text { (Valoró presencia de náuseas o vómito, disfagia, diarrea, peristaltismo, así mismo estado de } \\
\text { conciencia, capacidad de comprensión y de colaboración, glositis, estomatitis, limitaciones físicas o } \\
\text { psíquicas; Valoración de pares craneales glosofaríngeo e hipogloso, mucosa oral, motilidad de la } \\
\text { lengua, capacidad para movilizar el cuello, para el caso de medicamento vía oral) }\end{array}$ & $0 \%$ & NA \\
\hline $\begin{array}{l}\text { 6. Evaluó datos de laboratorio como pruebas de función renal y hepática, parcial de orina, cuadro } \\
\text { hemático, pruebas de coagulación, electrolitos, función tiroidea, si procedía }\end{array}$ & $0 \%$ & NA \\
\hline Analizó signos vitales & $14,3 \%$ & $16,3 \%$ \\
\hline Alistó el equipo completo de administración de medicamentos según era el caso & $100 \%$ & $95,3 \%$ \\
\hline 9. Se colocó los guantes limpios durante todo el proceso & $57,1 \%$ & $79,1 \%$ \\
\hline $\begin{array}{l}\text { 10. Envasó el medicamento aspirando con la jeringa la totalidad del fármaco sin tocar los bordes de la } \\
\text { ampolla }\end{array}$ & $100 \%$ & $79 \%$ \\
\hline 11. En caso de vial, desinfectó el tapón & $100 \%$ & $97,7 \%$ \\
\hline 12. Reconstituyó y envasó conservando la técnica aséptica & $100 \%$ & $95,3 \%$ \\
\hline 13. Aspiró con la jeringa la totalidad del fármaco & $71,4 \%$ & $100 \%$ \\
\hline 14. Explicó el procedimiento al paciente teniendo en cuenta sus condiciones psicológicas y culturales & $42,9 \%$ & $79,1 \%$ \\
\hline Informó sobre el tipo de medicamento, sus indicaciones y posibles efectos secundarios & $71,4 \%$ & $60,5 \%$ \\
\hline 16. Eliminó el aire de la jeringa & $100 \%$ & $93 \%$ \\
\hline $\begin{array}{l}\text { 17. Realizó la desinfección del puerto del equipo, o del buretrol o del tapón por donde administró el } \\
\text { medicamento }\end{array}$ & $71,4 \%$ & $81,4 \%$ \\
\hline $\begin{array}{l}\text { 18. Colocó o asistió al paciente para adoptar la posición más conveniente de acuerdo a la zona donde } \\
\text { aplicó el medicamento }\end{array}$ & $71,4 \%$ & $88,4 \%$ \\
\hline
\end{tabular}




\begin{tabular}{|c|c|c|c|}
\hline 19. & Determinó la zona de administración, para el caso de medicamento intramuscular o subcutáneo & $100 \%$ & $100 \%$ \\
\hline & $\begin{array}{l}\text { Realizó la limpieza de la piel adecuadamente, para el caso de medicamento intramuscular, } \\
\text { subcutáneo o intradérmico }\end{array}$ & $100 \%$ & $100 \%$ \\
\hline & $\begin{array}{l}\text { Evitó tocar la tableta, gragea o cápsula directamente con los dedos al administrar medicamentos vía } \\
\text { oral }\end{array}$ & $100 \%$ & $97,7 \%$ \\
\hline 22. & $\begin{array}{l}\text { En administración de medicamento por vía oral, ofreció la medicina y el agua al paciente y verificó } \\
\text { que la haya ingerido }\end{array}$ & $100 \%$ & $90,7 \%$ \\
\hline & $\begin{array}{l}\text { Administró el medicamento teniendo en cuenta las precauciones y técnica según la vía de } \\
\text { administración }\end{array}$ & $85,7 \%$ & $95,3 \%$ \\
\hline 24. & Dispuso los desechos en las canecas y recipientes destinados para cada uno de ellos & $71,4 \%$ & $93 \%$ \\
\hline 25. & Descartó agujas sin re enfundar en el guardián o recipiente según normas de bioseguridad & $71,4 \%$ & $53,5 \%$ \\
\hline 26. & Dejó al paciente cómodo manteniendo las medidas de seguridad & $71,4 \%$ & $88,4 \%$ \\
\hline 27. & Realizó lavado clínico de manos posterior a la realización de la administración de medicamentos & $28,6 \%$ & $18,6 \%$ \\
\hline 28. & Después de la administración valoró al paciente periódicamente en busca de reacciones adversas & $14,3 \%$ & $30,2 \%$ \\
\hline & $\begin{array}{l}\text { En el caso de administración intradérmica, a los } 20-30 \text { minutos valoró la pápula, mide tamaño y } \\
\text { comparó con induración de la inyección }\end{array}$ & $100 \%$ & $90,7 \%$ \\
\hline & $\begin{array}{l}\text { Elaboró el registro teniendo en cuenta: datos de identificación del paciente, fecha, hora, } \\
\text { medicamento, dosis, vía, nombre de quién administró el medicamento, tolerancia, reacciones } \\
\text { presentadas y otras observaciones que consideró pertinentes }\end{array}$ & $14,3 \%$ & $65,1 \%$ \\
\hline
\end{tabular}

Fuente: Autoras

Las prácticas que menos cumplieron los profesionales fueron: Valoración (antecedentes, examen físico, signos vitales, laboratorios) al paciente antes y después de la AM (5,7\%), Verificación de los correctos de la AM (14,3\%), Elaboración de los registros correspondientes (14,3\%), Lavado clínico de manos para el proceso de AM (antes y después) (35,8\%), Uso de elementos de protección personal $(57,1 \%)$, Explicación del procedimiento e información al paciente $(57,2 \%)$.

En cuanto a los técnicos auxiliares, las prácticas que menos cumplieron fueron: Lavado clínico de manos para el proceso de AM (antes y después) (26,8\%), Valoración (antecedentes y signos vitales) del paciente antes y después de la AM (31\%), Verificación de los correctos de la AM (37,2\%), Descarte seguro de residuos $(53,5 \%)$.

Las prácticas con mejor cumplimiento $(>90 \%)$, para ambos roles profesionales, fueron los enunciados relacionados con la técnica básica de AM, como preparación y aplicación del medicamento.

3.2.2. Lista de chequeo: Valoración de la seguridad del paciente en la administración de medicamentos

En promedio, el 29,7\% del personal no cumple con las prácticas de seguridad en la aplicación de los correctos de la AM.

Las prácticas de seguridad en la aplicación de los correctos en la AM, que más del 50\% del PE no cumple son: Usar al menos dos identificadores para confirmar al paciente antes de la AM, Identificar al paciente alérgico de forma notoria, Lavado clínico de manos previo a la AM, Realizar doble chequeo del cálculo en la preparación y AM potencialmente peligrosos o de estricta vigilancia, Registrar la hora de AM inmediatamente después de la administración de cada dosis, Registrar todos los eventos relacionados con la AM, Notificar en caso de incidentes relacionados con la terapia farmacológica, Mantener registros adecuados de la preparación de los medicamentos, Clarificar dudas acerca de la prescripción antes de la AM, Educar al paciente acerca del nombre del medicamento administrado, aspecto, justificación de la administración, frecuencia y efectos esperados, Orientar al paciente para identificar cualquier efecto indeseado del medicamento. Ver Tabla V 
Tabla V Prácticas en la aplicación de los correctos de la medicación del personal de enfermería

\begin{tabular}{|c|c|c|c|c|}
\hline Correcto & Valoración de la seguridad del paciente en la administración de medicamentos & Sí & NO & NA \\
\hline $\begin{array}{l}\text { Paciente } \\
\text { correcto }\end{array}$ & $\begin{array}{l}\text { 1. Usa al menos dos identificadores (nombre completo y número de historia } \\
\text { clínica) para confirmar al paciente antes de la administración de } \\
\text { medicamentos. }\end{array}$ & $24 \%$ & $76 \%$ & \\
\hline \multirow{5}{*}{$\begin{array}{l}\text { Medicamento } \\
\text { correcto }\end{array}$} & $\begin{array}{l}\text { 2. Verifica el nombre en la prescripción de medicamentos antes de } \\
\text { administrarlo. }\end{array}$ & $92 \%$ & $8 \%$ & \\
\hline & 3. Lleva a la cama solo los medicamentos prescritos para ese único paciente. & $88 \%$ & $12 \%$ & \\
\hline & Administra medicamento bajo orden verbal solo en caso de emergencia. & & & $100 \%$ \\
\hline & 5. $\quad$ Revisa si el paciente es alérgico al medicamento prescrito. & $56 \%$ & $44 \%$ & \\
\hline & $\begin{array}{l}\text { 6. Identifica al paciente alérgico de una forma notoria con una manilla, en la } \\
\text { historia clínica, alertando a todo el equipo profesional. }\end{array}$ & $12 \%$ & $62 \%$ & $26 \%$ \\
\hline \multirow{4}{*}{ Vía correcta } & 7. Identifica la vía de administración prescrita para el medicamento. & $100 \%$ & & \\
\hline & $\begin{array}{l}\text { 8. Revisa si la vía es técnicamente recomendada para la administración del } \\
\text { medicamento. }\end{array}$ & $96 \%$ & $4 \%$ & \\
\hline & 9. Se lava las manos, previo a la administración de medicamentos. & $34 \%$ & $66 \%$ & \\
\hline & 10. Usa técnica y material aséptico para la administración de medicamentos. & $86 \%$ & $14 \%$ & \\
\hline \multirow{3}{*}{ Hora correcta } & 11. Prepara el medicamento inmediatamente antes de la administración. & $80 \%$ & $20 \%$ & \\
\hline & 12. Administra el medicamento en la hora correcta. & $88 \%$ & $12 \%$ & \\
\hline & $\begin{array}{l}\text { 13. Ajusta los tiempos de administración de medicamentos a la rutina ya } \\
\text { establecida antes de la hospitalización. }\end{array}$ & $38 \%$ & & $62 \%$ \\
\hline \multirow{5}{*}{$\begin{array}{c}\text { Dosis } \\
\text { correcta }\end{array}$} & 14. Revisa cuidadosamente la dosis prescrita del medicamento. & $84 \%$ & $16 \%$ & \\
\hline & $\begin{array}{l}\text { 15. Coteja la velocidad de infusión, programación y operación de la bomba de } \\
\text { infusión continua con la prescripción. }\end{array}$ & $80 \%$ & $2 \%$ & $18 \%$ \\
\hline & $\begin{array}{l}\text { 16. Realiza doble chequeo del cálculo en la preparación y administración de } \\
\text { medicamentos potencialmente peligrosos o de estricta vigilancia. }\end{array}$ & $16 \%$ & $54 \%$ & $30 \%$ \\
\hline & $\begin{array}{l}\text { 17. Usa herramientas de medición estándar en la preparación de } \\
\text { medicamentos, para medir dosis precisas (ej. Jeringas milimétricas). }\end{array}$ & $100 \%$ & & \\
\hline & 18. Regresa las dosis de medicamento no administrado a la farmacia. & $4 \%$ & & $96 \%$ \\
\hline \multirow{4}{*}{$\begin{array}{l}\text { Registro } \\
\text { correcto }\end{array}$} & $\begin{array}{l}\text { 19. Registra la hora de administración del medicamento inmediatamente } \\
\text { después de la administración de cada dosis. }\end{array}$ & $38 \%$ & $62 \%$ & \\
\hline & $\begin{array}{l}\text { 20. Registra todos los eventos relacionados con la administración (ej. } \\
\text { Posposiciones, cancelaciones, paciente rehúsa, efectos secundarios, efectos } \\
\text { adversos). }\end{array}$ & $24 \%$ & $76 \%$ & \\
\hline & 21. Notifica en caso de incidentes relacionados con la terapia farmacológica. & & & $100 \%$ \\
\hline & $\begin{array}{l}\text { 22. Mantiene registros adecuados de la preparación de los medicamentos } \\
\text { (fecha y hora de la manipulación, concentración del medicamento, nombre } \\
\text { del responsable de la preparación y validación). }\end{array}$ & $48 \%$ & $52 \%$ & \\
\hline \multirow{2}{*}{$\begin{array}{l}\text { Educación } \\
\text { correcta }\end{array}$} & $\begin{array}{l}\text { 23. Clarifica dudas acerca de la prescripción antes de la administración del } \\
\text { medicamento (ej. Indicación de la medicación, dosis, "si es necesario", } \\
\text { unidades de medida usadas, forma farmacéutica, vía de administración). }\end{array}$ & $38 \%$ & $58 \%$ & $4 \%$ \\
\hline & $\begin{array}{l}\text { 24. Educa al paciente acerca del nombre del medicamento administrado, } \\
\text { aspecto (color y forma), justificación de la administración, frecuencia y } \\
\text { efectos esperados. }\end{array}$ & $38 \%$ & $62 \%$ & \\
\hline $\begin{array}{c}\text { Presentación } \\
\text { correcta }\end{array}$ & $\begin{array}{l}\text { 25. Revisa si el medicamento a administrar se encuentra en la forma } \\
\text { farmacéutica compatible con la vía de administración prescrita. }\end{array}$ & $98 \%$ & $2 \%$ & \\
\hline \multirow{2}{*}{$\begin{array}{l}\text { Respuesta } \\
\text { correcta }\end{array}$} & $\begin{array}{l}\text { 26. Orienta al paciente para identificar, si es posible, cualquier efecto indeseado } \\
\text { del medicamento. }\end{array}$ & $18 \%$ & $72 \%$ & $10 \%$ \\
\hline & $\begin{array}{l}\text { 27. Informa al prescriptor de todos los efectos diferentes a los esperados (en } \\
\text { intensidad y forma) por la administración de medicamentos. }\end{array}$ & $10 \%$ & $26 \%$ & $64 \%$ \\
\hline
\end{tabular}

Fuente: Autoras

Se resalta que, el 92\% del PE Verificó el nombre en la prescripción de medicamentos antes de administrarlo, el 100\% del PE Identificó la vía de administración prescrita, el 96\% Revisó si la vía es técnicamente recomendada para la administración, el 100\% Usó herramientas de medición estándar en la preparación de medicamentos y el $98 \%$ Revisó si el medicamento a administrar se encuentra en la forma farmacéutica compatible con la vía de administración.

Es importante denotar que, el 76\% del PE no usó al menos dos identificadores para confirmar al paciente antes de la $\mathrm{AM}$, el $76 \%$ no registró todos los eventos relacionados con la $\mathrm{AM}$, el $72 \%$ no orientó al paciente para identificar cualquier efecto indeseado del medicamento, el $66 \%$ no se lavó las manos antes de la AM, el $62 \%$ no educó al paciente acerca del nombre del medicamento administrado, 
aspecto, justificación de la administración, frecuencia y efectos esperados, el $62 \%$ no identificó al paciente alérgico de una forma notoria, el $62 \%$ no registró la hora de AM inmediatamente después de la administración de cada dosis, el $58 \%$ no clarificó las dudas acerca de la prescripción antes de la AM, el 54\% no realizó doble chequeo del cálculo en la preparación de medicamentos potencialmente peligrosos o de estricta vigilancia, y el 52\% no mantuvo registros adecuados de la preparación del medicamento.

El cumplimiento de las prácticas en la aplicación de los correctos de la medicación por rol profesional se dio así (ver Tabla VI):

Tabla VI Cumplimiento de las prácticas en la aplicación de los correctos de la medicación del personal de enfermería por rol profesional

\begin{tabular}{|c|c|c|c|}
\hline Correcto & Valoración de la seguridad del paciente en la administración de medicamentos & 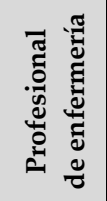 & 竞 \\
\hline $\begin{array}{l}\text { Paciente } \\
\text { correcto }\end{array}$ & $\begin{array}{l}\text { 1. Usa al menos dos identificadores (nombre completo y número de historia clínica) } \\
\text { para confirmar al paciente antes de la administración de medicamentos. }\end{array}$ & $0 \%$ & $27,9 \%$ \\
\hline \multirow{5}{*}{$\begin{array}{l}\text { Medicamento } \\
\text { correcto }\end{array}$} & Verifica el nombre en la prescripción de medicamentos antes de administrarlo. & $85,7 \%$ & $93 \%$ \\
\hline & Lleva a la cama solo los medicamentos prescritos para ese único paciente. & $85,7 \%$ & $88,4 \%$ \\
\hline & Administra medicamento bajo orden verbal solo en caso de emergencia. & NA & NA \\
\hline & Revisa si el paciente es alérgico al medicamento prescrito. & $42,9 \%$ & $58,1 \%$ \\
\hline & $\begin{array}{l}\text { 6. Identifica al paciente alérgico de una forma notoria con una manilla, en la historia } \\
\text { clínica, alertando a todo el equipo profesional. }\end{array}$ & $0 \%$ & $14 \%$ \\
\hline \multirow{4}{*}{ Vía correcta } & Identifica la vía de administración prescrita para el medicamento. & $100 \%$ & $100 \%$ \\
\hline & $\begin{array}{l}\text { 8. Revisa si la vía es técnicamente recomendada para la administración del } \\
\text { medicamento. }\end{array}$ & $100 \%$ & $95,3 \%$ \\
\hline & Se lava las manos, previo a la administración de medicamentos. & $42,9 \%$ & $30,2 \%$ \\
\hline & 10. Usa técnica y material aséptico para la administración de medicamentos. & $85,7 \%$ & $86 \%$ \\
\hline \multirow{3}{*}{ Hora correcta } & 11. Prepara el medicamento inmediatamente antes de la administración. & $100 \%$ & $76,7 \%$ \\
\hline & 12. Administra el medicamento en la hora correcta. & $85,7 \%$ & $88,4 \%$ \\
\hline & $\begin{array}{l}\text { 13. Ajusta los tiempos de administración de medicamentos a la rutina ya establecida } \\
\text { antes de la hospitalización. }\end{array}$ & $100 \%$ & $100 \%$ \\
\hline \multirow{5}{*}{$\begin{array}{l}\text { Dosis } \\
\text { correcta }\end{array}$} & 14. Revisa cuidadosamente la dosis prescrita del medicamento. & $85,7 \%$ & $83,7 \%$ \\
\hline & $\begin{array}{l}\text { 15. Coteja la velocidad de infusión, programación y operación de la bomba de } \\
\text { infusión continua con la prescripción. }\end{array}$ & $100 \%$ & $97,7 \%$ \\
\hline & $\begin{array}{l}\text { 16. Realiza doble chequeo del cálculo en la preparación y administración de } \\
\text { medicamentos potencialmente peligrosos o de estricta vigilancia. }\end{array}$ & $42,9 \%$ & $46,5 \%$ \\
\hline & $\begin{array}{l}\text { 17. Usa herramientas de medición estándar en la preparación de medicamentos, para } \\
\text { medir dosis precisas (ej. Jeringas milimétricas). }\end{array}$ & $100 \%$ & $100 \%$ \\
\hline & 18. Regresa las dosis de medicamento no administrado a la farmacia. & NA & $100 \%$ \\
\hline \multirow{4}{*}{$\begin{array}{l}\text { Registro } \\
\text { correcto }\end{array}$} & $\begin{array}{l}\text { 19. Registra la hora de administración del medicamento inmediatamente después de } \\
\text { la administración de cada dosis. }\end{array}$ & $14,3 \%$ & $41,9 \%$ \\
\hline & $\begin{array}{l}\text { 20. Registra todos los eventos relacionados con la administración (ej. Posposiciones, } \\
\text { cancelaciones, paciente rehúsa, efectos secundarios, efectos adversos). }\end{array}$ & $14,3 \%$ & $25,6 \%$ \\
\hline & 21. Notifica en caso de incidentes relacionados con la terapia farmacológica. & NA & NA \\
\hline & $\begin{array}{l}\text { 22. Mantiene registros adecuados de la preparación de los medicamentos (fecha y } \\
\text { hora de la manipulación, concentración del medicamento, nombre del } \\
\text { responsable de la preparación y validación). }\end{array}$ & $14,3 \%$ & $51,2 \%$ \\
\hline \multirow{2}{*}{$\begin{array}{l}\text { Educación } \\
\text { correcta }\end{array}$} & $\begin{array}{l}\text { 23. Clarifica dudas acerca de la prescripción antes de la administración del } \\
\text { medicamento (ej. Indicación de la medicación, dosis, "si es necesario", unidades } \\
\text { de medida usadas, forma farmacéutica, vía de administración). }\end{array}$ & $28,6 \%$ & $39,5 \%$ \\
\hline & $\begin{array}{l}\text { 24. Educa al paciente acerca del nombre del medicamento administrado, aspecto } \\
\text { (color y forma), justificación de la administración, frecuencia y efectos esperados. }\end{array}$ & $14,3 \%$ & $41,9 \%$ \\
\hline $\begin{array}{c}\text { Presentación } \\
\text { correcta }\end{array}$ & $\begin{array}{l}\text { 25. Revisa si el medicamento a administrar se encuentra en la forma farmacéutica } \\
\text { compatible con la vía de administración prescrita y no vencido. }\end{array}$ & $100 \%$ & $97,7 \%$ \\
\hline \multirow{2}{*}{$\begin{array}{l}\text { Respuesta } \\
\text { correcta }\end{array}$} & $\begin{array}{l}\text { 26. Orienta al paciente para identificar, si es posible, cualquier efecto indeseado del } \\
\text { medicamento. }\end{array}$ & $14,3 \%$ & $18,6 \%$ \\
\hline & $\begin{array}{l}\text { 27. Informa al prescriptor de todos los efectos diferentes a los esperados (en } \\
\text { intensidad y forma) por la administración de medicamentos. }\end{array}$ & $85,7 \%$ & $72,1 \%$ \\
\hline
\end{tabular}

Fuente: Autoras 
Los correctos de la medicación que mejor cumplieron el PE globalmente fue, Hora correcta (profesionales 95,2\% y técnicos auxiliares 88,4\%) y Presentación correcta (profesionales $100 \%$ y técnicos auxiliares $97,7 \%$ ). Las prácticas que menos cumplieron los profesionales por correcto de medicación fueron: i) Paciente correcto: Usar al menos dos identificadores para confirmar al paciente antes de la AM (0\%); ii) Medicamento correcto: Revisar si el paciente es alérgico al medicamento prescrito $(42,9 \%)$, Identificar al paciente alérgico de una forma notoria ( $0 \%)$; iii) Vía correcta: Lavado de manos previo a la AM (42,9\%); iv) Dosis correcta: Realizar doble chequeo del cálculo en la preparación y AM potencialmente peligrosos o de estricta vigilancia (46,5\%); v) Registro correcto: Registrar la hora de AM inmediatamente después de la administración de cada dosis $(14,3 \%)$, Registrar todos los eventos relacionados con la AM (14,3\%), Mantener registros adecuados de la preparación de los medicamentos (14.3\%); vi) Educación correcta: Clarifica dudas acerca de la prescripción antes de la AM (28,6\%), Educa al paciente acerca del nombre del medicamento administrado, aspecto, justificación de la administración, frecuencia y efectos esperados (14,3\%); vii) Respuesta correcta: Orienta al paciente para identificar cualquier efecto indeseado del medicamento $(14,3 \%)$.

Las prácticas que menos cumplieron los técnicos auxiliares por correcto de medicación fueron: i) Paciente correcto: Usar al menos dos identificadores para confirmar al paciente antes de la AM (27,9\%); ii) Medicamento correcto: Identificar al paciente alérgico de una forma notoria (14\%); iii) Vía correcta: Lavado de manos previo a la AM (30,2\%); iv) Dosis correcta: Realizar doble chequeo del cálculo en la preparación y AM potencialmente peligrosos o de estricta vigilancia (\%); v) Registro correcto: Registrar la hora de AM inmediatamente después de la administración de cada dosis (41,9\%), Registrar todos los eventos relacionados con la AM (25,6\%), Mantener registros adecuados de la preparación de los medicamentos (51,2\%); vi) Educación correcta: Clarifica dudas acerca de la prescripción antes de la administración del medicamento (39,5\%), Educa al paciente acerca del nombre del medicamento administrado, aspecto, justificación de la administración, frecuencia y efectos esperados (41,9\%); vii) Respuesta correcta: Orienta al paciente para identificar cualquier efecto indeseado del medicamento $(18,6 \%)$.

Se realizó análisis bivariado para establecer la asociación que existe entre los puntajes de los participantes en las dos listas de chequeo y se halló asociación fuerte entre los resultados (Coeficiente de correlación de Spearman 0,71), indicando la correlación entre el desempeño general en administración segura de medicamentos y la aplicación de los correctos de la medicación. En los grupos por rol profesional se encontró una prevalencia de no cumplimiento de las prácticas de administración segura de medicamentos de $27,9 \%$ para técnicos auxiliares y $42,9 \%$ para profesionales, con una razón de 0,65 en relación con el nivel de formación, lo que indica que la formación profesional no impacta al momento del cumplimiento de las prácticas de seguridad en la AM evaluadas.

\section{Discusión}

El ejercicio de prácticas de seguridad en AM en este estudio mostró mejor rendimiento entre personal técnico auxiliar (61\% de prácticas seguras), versus profesionales (49\% de prácticas seguras); sin embargo, ninguno de los dos grupos de PE alcanzó más del 90\% de prácticas seguras en AM, lo cual se corresponde con los hallazgos de Llapa-Rodríguez et al (2017) [15], que indican que los profesionales tiene adhesión a algunas prácticas de seguridad en la AM pero las debilidades encontradas comprometen toda la práctica segura.

Las prácticas seguras que más realiza el PE son, la preparación y aplicación correcta de medicamentos, cuyos porcentajes de cumplimientos superan el 90\%, y el mejor cumplimiento se da en aspectos básicos del proceso (envase y ejecución de la AM); siendo las prácticas de seguridad menos aplicadas por el PE: i) el lavado clínico de manos, que encuentra respaldo en otros estudios latinoamericanos relacionados al cumplimiento del protocolo de esta actividad, en los cuales el incumplimiento se da alrededor del 50\% [16,17]; ii) la verificación de los correctos de la medicación, que en otros estudios también ha mostrado incumplimiento con hasta $19 \%$ de errores asociados con estos[18]; y iii) la valoración del paciente antes y después, con cumplimiento por debajo del $40 \%$. 
Destaca que, de las nueve principales prácticas de seguridad en la AM, solo dos tengan más del $90 \%$ de cumplimiento por parte de ambos grupos del PE y que, en cinco de las nueve prácticas, los profesionales tengan bajos rendimientos en comparación con los técnicos auxiliares. Ver Figura 1.

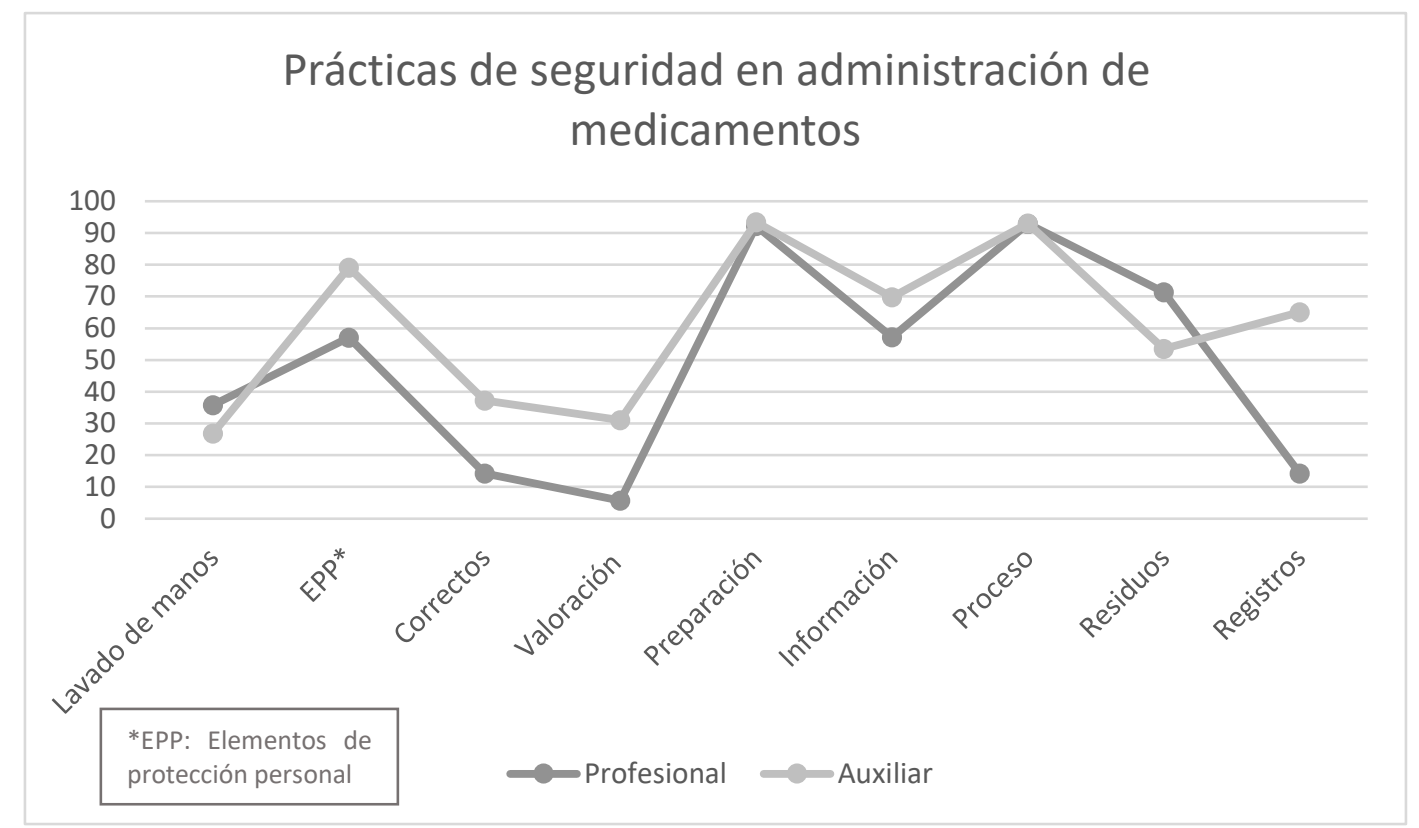

Figura 1 Prácticas de seguridad en administración de medicamentos, comparativo entre profesionales de enfermería y técnicos auxiliares de enfermería. Fuente: Autoras

Llama la atención que la Valoración (antecedentes, examen físico, signos vitales, laboratorios (antes y después de la administración de medicamentos)) que es una actividad predominante del profesional, sea desarrollada en más ocasiones por el técnico auxiliar (Figura 1); igualmente, los registros de enfermería deben ser elaborados idealmente por el profesional, sin embargo, fueron los técnicos auxiliares los que mayor cantidad de registros de AM, en relación con el proceso, realizaron (Figura 1). Estos resultados hallan acuerdo con el estudio de Pecina (2012) [19], que reporta déficit de registros y ausencia de registros de la valoración del paciente en la historia clínica. Además, a pesar de que no fueron bastante frecuentes, el $27 \%$ del PE no tiene prácticas seguras de eliminación de desechos y el $24 \%$ no utiliza elementos de protección personal.

El ejercicio de prácticas en la aplicación de los correctos de la medicación mostró mejor rendimiento entre personal técnico auxiliar $(67 \%)$, versus profesionales $(60,1 \%)$; sin embargo, se encontró prácticas de cumplimiento muy bajo $(<20 \%)$ en la aplicación de correctos de medicación en el PE. Aquellos correctos con menos del 50\% de cumplimiento fueron Paciente, Registro, Educación y Respuesta correcta para ambos grupos del PE [18]. Destaca que la práctica de Paciente correcto no cumplió con al menos el 50\% de aplicación por parte de ninguno de los dos grupos de PE.

El rendimiento de la aplicación de los correctos de la medicación, comparativo entre los dos grupos del PE, se puede ver en la Figura 2 


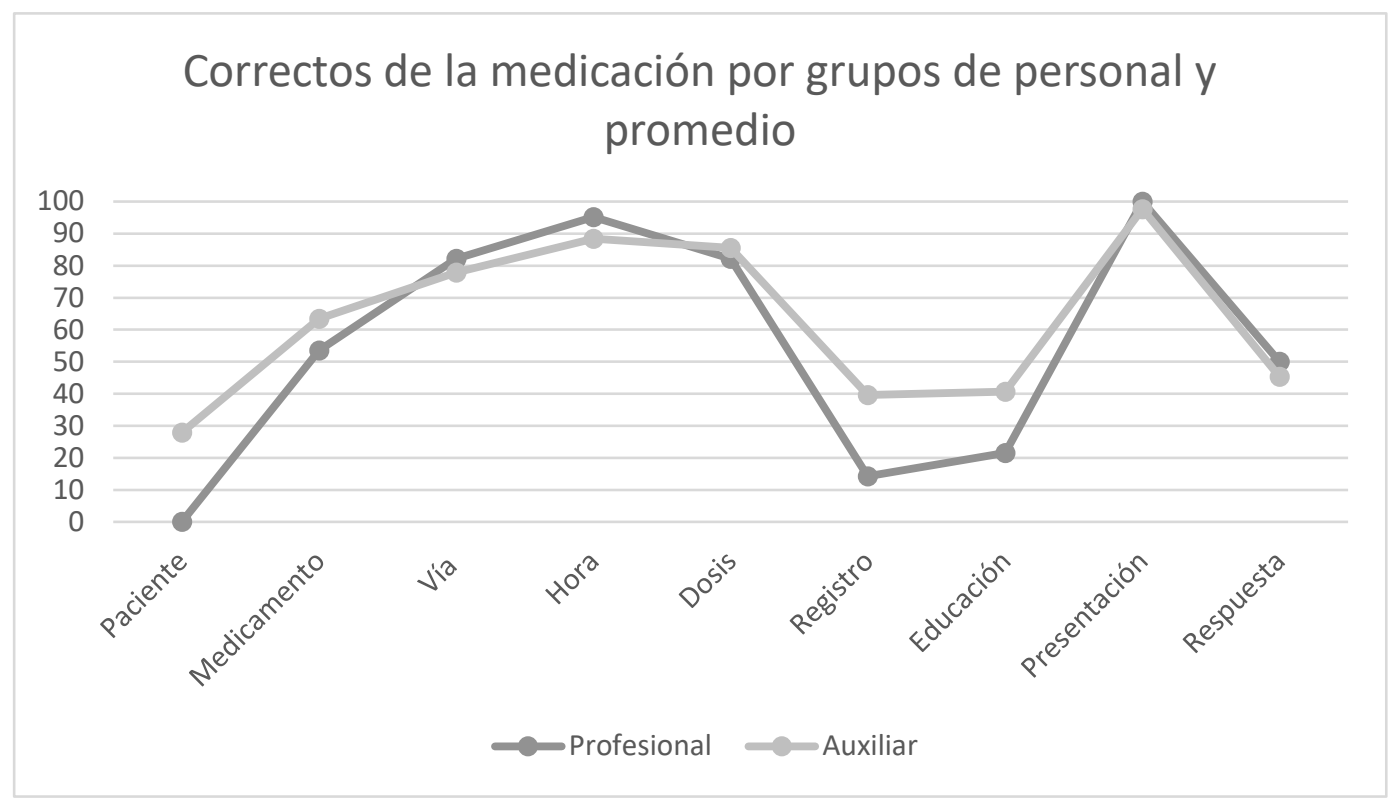

Figura 2 Rendimiento de aplicación de los correctos de la medicación, comparativo entre profesionales de enfermería y técnicos auxiliares de enfermería. Fuente: Autoras

Llama la atención que, de los cinco correctos de la medicación originales, todos tengan buen rendimiento, excepto Paciente correcto, alcanzando rendimiento tan bajo como $0 \%$ en el caso de los profesionales. Los otros cuatro correctos tienen rendimiento promedio, solo Hora correcta y Presentación correcta, rebasan el $90 \%$ de cumplimiento; los resultados se asemejan a los hallados en Brasil por Llapa-Rodríguez et al (2017) [15].

Los correctos de medicación con menor rendimiento promedio, además de Paciente correcto, son Registro correcto, Educación correcta y Respuesta correcta. Se debe tener en cuenta que otros correctos conocidos como Verificación de alergias, Fecha de vencimiento y Responsabilidad, están inmersos dentro de la verificación de Medicamento correcto, Presentación correcta y Respuesta correcta, respectivamente.

El Registro y la Educación son actividades que debe desarrollar predominantemente el profesional, sin embargo, se evidencia que lo realizaron en menor proporción que los técnicos auxiliares.

Por otro lado, al indagar por la autopercepción del nivel de conocimientos en administración segura de medicamentos (Tabla II), el PE manifestó tener conocimiento Bueno/Satisfactorio a Ideal/Muy satisfactorio, sin embargo, los resultados de aplicación de las prácticas de seguridad en AM tuvieron una prevalencia de no cumplimiento de $27,9 \%$ para técnicos auxiliares y $42,9 \%$ para profesionales. Este rendimiento en la evaluación de la seguridad de la AM puede estar relacionada con la delegación excesiva de la actividad de AM por parte del profesional [20], lo que puede impactar en la discontinuidad de la praxis para el profesional en favor de la habitualidad con la AM para el técnico auxiliar; y aunado a ello, aunque los dos grupos del PE eran similares en su reporte de experiencia laboral en área asistencial, sí se observa que el 76,8\% de los técnicos auxiliares reportó que el servicio dónde ha laborado la mayor parte del tiempo fue en servicios de urgencias o UCI versus los profesionales (42,9\%) (Ver Tabla II).

Los resultados de este estudio invitan a mantener al personal bajo actualizaciones continuas del conocimiento (farmacología y seguridad) que incluyan talleres para la praxis; al respecto, los protocolos de seguridad en la prescripción, uso y AM recomiendan que las instituciones de salud brinden a sus trabajadores educación permanente, así como capacitación anual para la ejecución segura de esta práctica [15], ya que el entrenamiento estandarizado reduce significativamente la probabilidad de errores y favorece su interceptación antes de que el evento llegue al paciente y se convierta en EM [15,21]. Teniendo en cuenta, además, que en este estudio los profesionales reportaron menor porcentaje de actualizaciones en administración segura de medicamentos $(28,5 \%)$ frente a lo reportado por técnicos auxiliares $(37,2 \%)$, lo cual también pudo influir en las prevalencias reportadas. 
Se debe considerar que el inadecuado conocimiento y la omisión de las prácticas seguras y de los correctos de medicación puede resultar en EM, que son asociadas a las habilidades (prácticas) del PE [22-24] y que son frecuentes debido a que no existe ningún filtro que pueda detectar o prevenir los errores, siendo el mismo PE quien se autocontrola [25] y controla la aparición de EM [26]; en este estudio el $14,3 \%$ de los profesionales reportó que autopercibe su conocimiento en administración segura de medicamentos como Malo/Insatisfactorio. La AM es una actividad de enfermería que requiere un proceso reflexivo e implica una responsabilidad, adicional a conocimientos farmacológicos y técnicos [23], pues a partir de los EM las consecuencias son asumidas por el paciente.

\section{Conclusiones}

El PE, en este estudio, mostró cumplimiento de prácticas seguras en AM alrededor de la media de cumplimiento, con un rendimiento similar en ambos grupos del PE (Razón de prevalencias 0,65) y en ambas listas de chequeo (Coeficiente de Spearman 0,71). Las prácticas seguras que más realizaron los dos grupos del PE se relacionan con las actividades básicas en la AM como la preparación y aplicación de los mismos, sin embargo, la seguridad del paciente puede verse afectada por el incumplimiento de otras prácticas en la AM como, la valoración del paciente (antes y después de la AM) y la educación e información al paciente, que fueron las actividades menos ejecutadas por el PE.

\section{Recomendaciones}

Se espera que estos resultados subsidien la gestión del PE, con el fin de diseñar estrategias que minimicen las fragilidades identificadas, con el fin de hacer seguro el proceso de AM. Asimismo, los resultados ofrecen una herramienta importante para las instituciones asistenciales y académicas, como representación de la cultura de seguridad del paciente, fomentando así futuras discusiones que promuevan la construcción de una cultura de seguridad.

Se deben realizar nuevas investigaciones con el fin de comprender mejor los factores relacionados con las vulnerabilidades encontradas, lo cual permitirá un análisis amplio sobre el tema en las instituciones asistenciales, académicas y en la comunidad científica.

Conflictos de Intereses: Las autoras no declaran conflicto de intereses.

\section{Referencias Bibliográficas}

1. Organización Mundial de la Salud OMS. Seguridad del paciente [nota descriptiva]. 2019 [citado 2021 mar 10]. Disponible en: https://www.who.int/es/news-room/fact-sheets/detail/patient-safety

2. Figueiredo MB, Guedes CL, Thibau FF, dos Reis CA, da Silva DA, Leite AC. Seguridad en la administración de medicamentos: investigación sobre la práctica de enfermería y circunstancias de errores. Enfermería glob. [internet] 2019; 56: 19-31. Disponible en: http://scielo.isciii.es/pdf/eg/v18n56/1695-6141-eg-18-5619.pdf

3. Yesilyaprak T, Demir KF. The relationship between surgical intensive care unit nurses' patient safety culture and adverse events. Nurs Crit Care [internet] 2021; 1-9. Available in: https://onlinelibrary.wiley.com/doi/10.1111/nicc.12611. DOI: https://doi.org/10.1111/nicc.12611

4. Isaacs AN, Ch'ng K, Delhiwale N, Taylor K, Kent B, Raymond A. Hospital medication errors: a cross sectional study. Int J Qual Health Care [internet]. 2020; 33(1) Available in: https:/onlinelibrary.wiley.com/doi/epdf/10.1111/nicc.12611. DOI: https://doi.org/10.1093/intqhc/mzaa136

5. Ministerio de Salud de Colombia. Mejorar la seguridad en la utilización de medicamentos. Paquetes instruccionales. Guía técnica: Buenas prácticas para la seguridad del paciente en la atención en salud. Versión 2.0. 2018. $133 p$.

Disponible en: 
https://www.minsalud.gov.co/sites/rid/Lists/BibliotecaDigital/RIDE/DE/CA/seguridad-en-la-utilizacionde-medicamentos.pdf

6. Mekonen EG, Gebrie MH, Jemberie SM. Magnitude and associated factors of medication administration error among nurses working in Amhara Region Referral Hospitals, Northwest Ethiopia. J Drug Assess [internet] 2020; 9(1): 151-158. Available in: https://www.tandfonline.com/doi/full/10.1080/21556660.2020.1841495. DOI: https://doi.org/10.1080/21556660.2020.1841495

7. Organización Mundial de la Salud - Organización Panamericana de la Salud. Estrategia y plan de acción para mejorar la calidad de la atención en la prestación de servicios de salud 2020-2025. 57 Consejo Directivo. 71르 Sesión del Comité Regional de la OMS para las Américas. Estados Unidos. 2019: 34 pp. Disponible en internet en: https://www.paho.org/hq/index.php?option=com_docman\&view=download\&alias=49721-cd57-12-sstrategia-pda-calidad-atencion\&category_slug=cd57-es\&Itemid=270\&lang=es

8. Iloh GU, Emeka EA, Ikwudinma AO, Amadi AN. Patient safety in a Resource-constrained context: a crosssectional study of a experience, drivers, barriers and preventive measures for safety incidents and accidents amongst medical doctors in South-east Nigeria. Niger Postgrad Med J [internet] 2020; 27(3): 202-208. Available in: https://www.npmj.org/article.asp?issn=11171936; year=2020; volume=27;issue $=3$; spage $=202$; epage $=208$; aulast $=$ =Iloh

9. Escrivá GJ, Brage SR, Fernández GJ. Medication errors and drug knowledge gaps among critical-care nurses: a mixed multi-method study. BMC Health Serv. Res. [internet] 2019; 19(640). Disponible en: https://bmchealthservres.biomedcentral.com/articles/10.1186/s12913-019-4481-7.

DOI: https://doi.org/10.1186/s12913-019-4481-7

10. Macías M, Solís L. Errores en la administración de medicación en un servicio de urgencias: conocer para disminuir el riesgo. Rev Esp Salud Pública [internet]. 2018; 92 (8): 1 -8. Disponible en: http://scielo.isciii.es/pdf/resp/v92/1135-5727-resp-92-e201806038.pdf

11. Plevová I, Jarosová D, Janíková E, Zeleníková R, Mynaríková E, Polanská A. Rationed nursingcare as one of the indicators of the occurrence of medication errors. Vnitr Lek [internet] 2020; 66(7): 31-38. Available in: https://pubmed.ncbi.nlm.nih.gov/33380132/

12. Bautista LM. Vejar LY. Pabón MR. Moreno JJ. Fuente L. León KY et al. Grado de adherencia al protocolo de registros clínicos de Enfermería. Rev Cuid. [internet] 2016; 7(1): 1195-203. Disponible en: https://www.redalyc.org/articulo.oa?id=359543375007

13. Marín A. Bonilla A. Rojas Z. Guarnizo M. Manual para la administración de medicamentos desde el proceso de atención de enfermería: un enfoque para la seguridad del paciente. $1^{\underline{a}}$ ed. Ed. Universidad El Bosque. Bogotá D.C. 2018. 20 pp. Disponible en: https://www.unbosque.edu.co/sites/default/files/201809/Manual\%20para\%20la\%20administracio\%CC\%81n\%20de\%20medicamentos.pdf

14. Rebouças P.Elisangela F. Moura M. Paz S. Fontenele R. de Almeida P. Medication administration safety assessment tool: Construction and validation. Rev. Bras. Enferm. 2019; 72(2): 329-36. Available in: https://www.scielo.br/j/reben/a/tcsswCDngjtPvMcvPnFRkjb/?lang=en.

DOI: http://dx.doi.org/10.1590/0034-7167-2018-0340

15. Llapa-Rodríguez E. Lobo L. Oliveira M. Albuquerque J. Currie L. Safe patient care in the preparation and administration of medicines. Rev Gaúcha Enferm. 2017; 38(4):e2017-0029. Available in: https://www.scielo.br/j/rgenf/a/mfp3NmJBdnNjnC6VVq8tpLr/?lang=en.

DOI: http://dx.doi.org/10.1590/1983-1447.2017.04.2017-0029.

16. Bloch Y. Acuña G. Oliveira H. Orué P. Cumplimiento del protocolo de Lavado de manos por profesionales de enfermería en un servicio de salud de la ciudad de Encarnación, marzo-julio de 2019. Mem. Inst. Investig. Cienc. Salud. 2020; 18(2): 6-11. Disponible en: http://scielo.iics.una.py/pdf/iics/v18n2/1812-9528iics-18-02-6.pdf. DOI: http://dx.doi.org/10.18004/mem.iics/1812-9528/2020.018.02.06

17. Salcedo-Cifuentes M. Ordoñez-Hernández C. Calvo-Soto A. Cumplimiento de una estrategia de higiene de las manos en ambientes asistenciales. Investig Enferm Imagen Desarr. 2020; 22. Disponible en: https://revistas.javeriana.edu.co/files-articulos/IE/22\%20(2020)/145263339003/. DOI: https://doi.org/10.11144/Javeriana.ie22.cehm

18. Domínguez M. Pérez J. Soto M. Eficacia de la práctica de enfermería en la administración de medicamentos. Rev CONAMED. 2015; 20(1): S35-S40. Disponible en: https://www.medigraphic.com/pdfs/conamed/con2015/cons151f.pdf 
19. Pecina R. Registros de Enfermería y la Aplicación del Proceso enfermero en el Área de recuperación quirúrgica. Desarrollo Cientif Enferm. 2012; 20(3): 83-86. Disponible en: http://www.indexf.com/dce/20pdf/20-083.pdf

20. Ortega M. Jiménez A. Situación actual de Enfermería en Colombia: Una reflexión. Rev Cuidado y $\begin{array}{lllll}\text { ocupación } & \text { humana. } & \text { 2020; } & \text { 28-38. } & \text { Disponible }\end{array}$ http://revistas.unipamplona.edu.co/ojs_viceinves/index.php/COH/article/view/4448/2612

21. Browne F, Hannigan B, Harden J. A realist evaluation of a safe medication administration education programme. NEDT [internet]. 2021; 97: 1 - $\quad 9 . \quad$ Available in: https://www.sciencedirect.com/science/article/pii/S0260691720315355?via\%3Dihub. DOI: https://doi.org/10.1016/j.nedt.2020.104685

22. Craig SJ, Kastello JC, Cieslowski BJ, Rovyak V. Simulation strategies to increase student clinical competence in safe medication administration practices: A quasi-experimental study. Nurse Educ Today. [internet] 96(104605) Available

in: https://www.sciencedirect.com/science/article/abs/pii/S0260691720314556?via\%3Dihub. DOI: https://doi.org/10.1016/j.nedt.2020.104605

23. Ali M, Saifan A, Alrimawi I, Atout M. Nurses' perceptions toward factors that cause medication errors in Jordan: A qualitative study. Perspect Psychiatr care [internet] 2020; 1-8. Available in: https://onlinelibrary.wiley.com/doi/10.1111/ppc.12707

24. Schroers G, Gunberg RJ, Moriarty H. Nurses' perceived causes of medication administration errors: a qualitative systematic review. JT Comm J Qual Patient Saf [internet] 2021; 47(1): 28-53. Available in: https://www.sciencedirect.com/science/article/abs/pii/S1553725020302476?via\%3Dihub. DOI: https://doi.org/10.1016/j.jcjq.2020.09.010

25. Escrivá GJ, Aparisi SA, Brage SR, Fernández GJ. Medication errors and risk areas in critical care unit. J Adv Nurs. [internet] 2020; 00: 1-10. Available in: https://onlinelibrary.wiley.com/doi/10.1111/jan.14612. DOI: https://doi.org/10.1111/jan.14612

26. Mardani A, Griffiths P, Vaismoradi M. The role of the nurse in the management of medicines during transitional care: a systematic review. J Multidiscip Healthc. [internet] 2020; 13: 1347-1361. Available in: https://www.dovepress.com/the-role-of-the-nurse-in-the-management-of-medicines-during-transitionpeer-reviewed-fulltext-article-JMDH

(C) 2021 por los autores; Esta obra está sujeta a la licencia de Reconocimiento 4.0 Internacional de Creative Commons. Para ver una copia de esta licencia, visite http://creativecommons.org/licenses/by-nc-nd/4.0/. 